\title{
The tRNA world
}

\author{
ROLAND K. HARTMANN, ${ }^{1}$ MARIO MÖRL, ${ }^{2}$ and MATHIAS SPRINZL ${ }^{3}$ \\ ${ }^{1}$ Philipps-Universität Marburg, Institut für Pharmazeutische Chemie, 35037 Marburg, Germany \\ ${ }^{2}$ Max Planck Institute for Evolutionary Anthropology, 04103 Leipzig, Germany \\ ${ }^{3}$ Laboratorium für Biochemie, Universität Bayreuth, 95440 Bayreuth, Germany
}

The 20th International tRNA Workshop 2003 was held in Kloster Banz, an old monastery with a fateful history dating back to the eighth century. On the scientific time scale, the International tRNA Workshop has a history almost as long and eventful as Kloster Banz, with its first celebration in Cambridge, UK, in 1969.

Transfer RNAs are unique. Not only are they the beststudied RNA structures, they are also a treasury of nucleoside modifications. They are the crucial link between deciphering of genetic code and protein biosynthesis, interacting with a pleiotropy of other cellular macromolecules, such as synthetases, processing and modification enzymes, translation factors, and, remarkably, with the only two genuine RNA enzymes of extant organisms: RNase P and the ribosome. Being present in all kingdoms of life as well as eukaryotic organelles, tRNAs are invaluable keys to the understanding of how the genetic code and the protein synthesis machinery coevolved. A common perception during the 20th tRNA Workshop was formulated by Knud $\mathrm{H}$. Nierhaus, who chaired the last session on translation: We are close to speaking the same language, which means there is increasing mutual interest and understanding among those specialized in tRNA bioinformatics, structure, evolution, modification, processing, charging, transport, or ribosome function.

The first session ("tRNA Genomics, tRNA Structure, tRNA in Disease") was opened by Sharief Barends, describing recent advances toward understanding the role of tRNA-like structures (TLS) at the $3^{\prime}$ end of plant virus genomes, which has been a heavily disputed area of research for the past two decades. Turnip yellow mosaic virus, for example, uses its valylated $3^{\prime}$ TLS as a bait to attract host ribosomes to internal initiation sites in the viral mRNA. Brome mosaic virus carries an enlarged TLS at the $3^{\prime}$ ends of its three genomic RNAs with determinants for tyrosylation. The TLS is required for translation of viral RNAs, but

Reprint requests to: Mathias Sprinzl, Laboratorium für Biochemie, Universität Bayreuth, 95440 Bayreuth, Germany; e-mail: mathias.sprinzl@ uni-bayreuth.de; fax: +49/921/55 2432 .

Article and publication are at http://www.rnajournal.org/cgi/doi/ 10.1261/rna.5240904. apparently does not direct tyrosine incorporation into viral proteins.

Investigation of the cellular and molecular defects caused by pathogenic point mutations in mitochondrial tRNAs was one of the prominent themes in Banz, and was addressed in talks by Catherine Florentz, Shana O. Kelley, Yohei Kirino, and Takashi Nagaike. A quantitative two-dimensional electrophoretic analysis performed for cells with mitochondria carrying a point mutation in tRNA ${ }^{\text {Lys }}$ (A8344G; MERRF syndrome) has revealed that a number of nuclear-encoded proteins were either up- or down-regulated in the mutant cells. These findings evidence an intimate interdigitation of mitochondrial and nuclear genome expression patterns, which we are only beginning to understand. Why do pathogenic mitochondrial mutations cluster in human mitochondrial tRNA ${ }^{\text {Ile }}$ and tRNA ${ }^{\text {Leu }}$ (UUR)? Evidently those human mitochondrial tRNAs with unique sequences and weak tRNA architecture are most vulnerable to mutation-induced conformational destabilization. Kimitsuna Watanabe and coworkers have established a mammalian mitochondrial in vitro translation system, a remarkable achievement by itself, with the goal to dissect the defects of mutant tRNAs in mitochondrial translation. A key observation is the absence of the taurine wobble modification in several mutant tRNAs associated with human diseases, which apparently causes defects in codon recognition and discrimination. Takashi Nagaike reported that two human mitochondrial tRNA mutations (A4317G in $\mathrm{mt} \mathrm{tRNA}^{\text {Ile }}$ and A10044G in $\mathrm{mt} \mathrm{tRNA}^{\text {Gly }}$ ) impaired CCA addition to these tRNAs by the human mitochondrial CCA-adding enzyme, a defect which may contribute to mitochondrial dysfunction.

Noteworthy and related to disease is the finding that alternative splice variants or proteolytic fragments of mammalian TrpRS or TyrRS are involved in the regulation of endothelial cell signaling processes, a topic covered by Karla Ewalt and Xiang-Lei Yang. Remarkably, the proteolytic core fragments (mini-TyrRS, mini-TrpRS) of TrpRS and TyrRS have pro-angiogenic (mini-TyrRS) and anti-angiogenic (mini-TrpRS) activities, thus illustrating the "yin-yang" in the regulation of angiogenesis.

The talks by Jeffrey Wong and David Ardell illustrated the revival of the almost forgotten tRNA as an evolutionary 
marker in the new genomics era. Jeffrey Wong guided us on an inspiring tour into the depth of tRNA evolution, starting from the TyrRS-TrpRS paralog pair whose evolutionary distance within an organism was found to be a measure of the extent of evolution within this organism. Sixty different genomes were scrutinized for the existence of closely related "alloacceptor" tRNAs that accept dissimilar amino acids and are likely paralogs derived by gene duplication. The analysis identified, for example, extremely conserved tRNA $^{\text {Phe }}-$ tRNA $^{\text {Tyr }}$ pairs with up to $94 \%$ sequence identity in some archaeal species. The lowest alloacceptor distances were observed in Methanopyrus kandleri and Aeropyrum pernix. Taking this as a measure for the primitiveness of the tRNA genotype, the last universal common ancestor (LUCA) may be placed betweeen the branches leading to these two Archaea in the universal tRNA phylogenetic tree.

The talk by David Ardell was one of the examples proving that bioinformatics can indeed be communicated to a primarily nonbioinformatic audience. The author defined identity families of bacterial tRNA ("tFAMs"), starting from the Sprinzl database (new version at http://www.tRNA.unibayreuth.de) of empirically characterized tRNAs, with the goal of studying the diversity of tRNA sequence identity rules in bacterial genomes. The program tFAM (freeware available soon; a link will be announced at http://www. eva.mpg.de/genetics/tRNA/) allows to classify initiator, suppressor, and pseudo-tRNAs with high confidence. Interestingly, tFAM analysis revealed abnormal tRNA ${ }^{\mathrm{His}}$ species in some branches of $\alpha$-proteobacteria, namely in the Rhizobiales and Caulobacter. These tRNA ${ }^{\text {His }}$ species lack the potential to form an 8-bp acceptor stem due to the fact that the identity of $n t-1$ is not $G$ and the discriminator is $A$ instead of C. Moreover, in Rhizobiales and Caulobacter, the active site of HisRS covaries perfectly with $\mathrm{tRNA}^{\mathrm{His}}$ variation, lacking Q164 as eukaryotic HisRS enzymes do and thus providing further evidence for lateral gene transfer of HisRS from eukarya to bacteria.

To date, more than 80 modified nucleosides in tRNAs have been identified and characterized. Transfer RNA modification enzymes have attracted attention as novel drug targets and represent treasure chests toward the understanding of RNA-protein interactions. The comeback of the tRNA modification field was one of the common perceptions in Banz, particularly with respect to the novel structures of tRNA modification enzymes as well as the elaborate strategies developed to uncover such enzymes. The new and exciting structures presented by Shigeyuki Yokoyama, Joseph Watts, and Louis Droogmans included (1) TrmH catalyzing 2'-O-methylation of $\mathrm{G}_{18}$ in the $\mathrm{D}$ loop, a modification that is introduced into all tRNAs in the thermophilic bacterium Thermus thermophilus, a particular feature of the central catalytic domain is its knotted structure; (2) the related TrmD from Escherichia coli, methylating $G_{37}$ in tRNAs containing the sequence $G_{36} / G_{37}$, whose catalytic site architecture depends on homodimerization;
(3) archaeosine tRNA-guanine transglycosylase modifying $\mathrm{G}_{15}$ in the D arm of many archaeal tRNAs and binding its substrates in a dramatically rearranged, so-called $\lambda$ conformation of tRNA; and (4) the Pyrococcus abyssi TrmI methytransferase catalyzing formation of 1-methyladenosine $\left(\mathrm{m}^{1} \mathrm{~A}\right)$ not only at tRNA position 58 , but also at 57 as an intermediate in the biosynthesis of 1-methylinosine at this location.

About 50\% of RNA modification genes have not been identified yet. Valérie de Crécy-Lagard illustrated the scientific power resulting from a "liaison" between bioinformatics and functional genetics. In her comparative genomics approach, candidates for missing tRNA modification genes are explored, combining homology searches, phylogenetic occurrence of the modification, gene clustering, and prediction of the kind of enzyme activity required. Candidate genes are then tested by chromosomal knockout mutations, for example, in Acinetobacter calcoaceticus, a gram-negative proteobacterium that is easy to manipulate genetically. By this strategy, the missing links in the synthesis of the queuine precursor, 7-aminoethyl 7-deazaguanine (preq1) from GTP were identified. Along the same lines, Tsutomu Suzuki and colleagues pursued an approach named ribonucleome analysis, which combines genetics and ion trap mass spectrometry (LC/MS). The profile of tRNA modifications (analyzed by LC/MS) of wild-type (E. coli, Bacillus subtilis, or yeast) cells is compared with the profile of cells carrying deletions of (nonessential) chromosomal segments. If specific modifications are absent in such a mutant strain, candidate genes for tRNA modification enzymes within the deletion region are supplied on a plasmid to test restoration of tRNA modification. A variant approach was applied to identify essential genes, such as that encoding the enzyme responsible for the introduction of lysidine (L) into the anticodon of tRNA. Here, candidate genes were selected by homology searches in genomes of various bacteria possessing the lysidine modification, and then tested for essentiality as well as a defect in lysidine modification in corresponding conditionally lethal mutant strains. This approach identified the bacterial tilS gene (tRNA ${ }^{\text {Ile }}$ lysidine synthetase).

The "tRNA Processing, Transport, and Channeling" session was opened by Dirk Görlich, discussing the function of the nuclear export machinery in counteracting the constant leakage of cytoplasmic proteins into the nuclear compartment. For example, Exportin 6 excludes actin from the nucleus and Exportin 5 excludes aa-tRNA-eEF1A-complexes from the nucleus. George Simos and coworkers dissected the role of Arclp in the nucleo-cytoplasmic trafficking of tRNA and the tRNA aminoacylation machinery. The yeast tRNA binding protein Arclp associates with MetRS and GluRS, facilitating their catalytic activity and restricting their presence to the cytoplasmic compartment. Applying the power of yeast genetics, such as the construction and analysis of yeast mutant strains expressing MetRS and 
GluRS variants lacking the noncatalytic domains conferring association with Arclp, the authors could reach the following conclusion: the Arclp-MetRS-GluRS complex enters the nucleus, but it is-after association with its tRNA cargo-efficiently exported via an Arclp- and Xpolp-dependent mechanism.

Several aminoacyl-tRNA synthetases carry an EMAPII RNA-binding domain that was originally discovered in endothelial-monocyte-activating polypeptide II. Marie-Pierre Golinelli reported that such a binding domain is also present in some auxiliary proteins that are associated with aminoacyl-tRNA synthetases in multienzyme complexes in eukaryotic cells. Examples for such proteins are the human p43 and the above-mentioned Arcp1p from yeast. Both of them carry an EMAPII-like domain at their C-terminus, mediating nonspecific RNA interactions. Interestingly, when the EMAPII (and middle) domains of p43 and Arclp are transferred to the catalytic core fragment of rice MetRS, they function as tRNA-binding domains; such fusion proteins are then capable of complementing a yeast strain deficient in Arcp1 and MetRS. It was further demonstrated by genetic complementation that human $\mathrm{p} 43$ can restore the growth rate of a yeast $\operatorname{arcl}^{-}$strain although p43 cannot interact with yeast MetRS and GluRS. Human p43 even restored cell viability of a double mutant $\operatorname{arc}^{-} l o s^{-}$, which is also defective in tRNA transport. These findings indicate that, in addition to its role as a cofactor for yeast MetRS and GluRS, Arclp may also be involved in a tRNA transport/ localization pathway, where it can be replaced by the human p43 protein.

What has been the news from the tRNA processing field? In eukaryotes, the $5^{\prime}$-endonuclease RNase $\mathrm{P}$ has a sister protein endonuclease, named RNase Z or $3^{\prime}$-tRNase, which cleaves tRNA $3^{\prime}$ precursors after the discriminator nucleotide. Anita Marchfelder reported that RNase Z, originally discovered in Eukarya and Archaea, also exists in B. subtilis encoded by the $y q j K$ gene, now renamed $r n z$. Apparently, B. subtilis RNase Z acts selectively on tRNA $3^{\prime}$ precursors that do not have a CCA terminus encoded in their genes. Remarkably, the gene for RNase $\mathrm{Z}$ of Drosophila melanogaster encodes both the nuclear and mitochondrial versions of the enzyme.

Regarding RNase $\mathrm{P}$, the structures of three bacterial RNase P proteins are known, but the RNA community has long been waiting for a structure including the P RNA subunit. As a first milestone, an X-ray structure of the specificity domain of B. subtilis P RNA was solved, resulting from a collaborative effort of Alfonso Mondragón's and Tao Pan's groups. Tao Pan took us on an excursion into the architecture of this domain, which includes a large nonhelical, well-structured module rich in metal-ion-binding sites. Furthermore, the structure has brought us close to the point of understanding how this RNA domain recognizes the $\mathrm{T}$ arm module of tRNAs exclusively via non-WatsonCrick tertiary interactions.
Bacterial RNase P ribozymes have the feature to be rather prone to "miscleavage" if single active site contacts are disconnected. This is biologically meaningful because two bacterial precursor tRNAs (ptRNA ${ }^{\text {His }}$ and $\mathrm{ptRNA}^{\mathrm{Sec}}$ ) have to be processed at a site shifted by $1 \mathrm{nt}$ to generate a mature tRNA product with an 8-bp-long instead of a 7-bp-long acceptor stem. Using genetics (mutations) and chemogenetics (exchange of single functional groups) as tools, Ema Kikovska reported new findings on the interplay of functional elements at the scissile phosphodiester in E. coli P RNA ribozyme-substrate complexes.

David Engelke and coworkers have found in yeast that both RNase P and ptRNAs reside mainly in the nucleolus, rather than the nucleoplasm, suggesting cooperative regulation of tRNA and ribosomal biogenesis. Likewise, multiple tRNA gene families and RNA polymerase III seem to be concentrated in the nucleolus as well. This, combined with the finding that yeast RNase $\mathrm{P}$ interacts with a subunit (Bdp1p) of transcription factor IIIB, suggests a model according to which both synthesis and early processing of ptRNA are coordinated at the nucleolus, along with ribosome biogenesis.

Two exciting sessions were dedicated to aminoacyl-tRNA synthetases ("Structure and Evolution" and "Mechanism and Novel Function"). The field of aminoacyl-tRNA synthetases has diversified immensely and reached a level of informational detail in terms of structures, mechanisms, novel functions, and evolutionary cross-relations, which makes it impossible to appreciate all these contributions as part of this space-limited conference report.

Prominent themes in the synthetase field have been the evolution of AspRS/AsnRS and GluRS/GlnRS enzymes, as well as the structural basis for discrimination or nondiscrimination against RNA $^{\text {Asn }}$ and tRNA ${ }^{\text {Gln }}$ by different AspRS and GluRS enzymes, respectively. The labyrinthine paths of synthetase evolution are illustrated by Hubert Dominique Becker's presentation. All Archaea with completed genome sequences that possess a gene for AsnRS also encode a paralog named AsnRS2 that, however, lacks the $\mathrm{N}$ terminal anticodon binding domain of AsnRS enzymes. In contrast to AsnRS, the AsnRS2 enzyme is unable to charge tRNA $^{\text {Asn }}$, but instead catalyzes the conversion of aspartic acid to asparagine. Phylogenetic analysis suggests a scenario where an AspRS progenitor in the eukaryal-archaeal kingdom was first duplicated, giving rise to AsnRS; a second later gene duplication within the Archaea generated AsnRS2. AsnRS2 apparently represents a link in the evolution of bacterial asparagine synthetase A that likely derived from archaeal AsnRS2 acquired by lateral gene transfer.

A tessera for the reconstruction of GlnRS evolution was provided by Tamara L. Hendrickson. Phylogenetic analyses predict that GlnRS arose from glutamyl-tRNA synthetase (GluRS) via gene duplication with subsequent evolution of specificity. GlnRS is an exception to the universality of the translation apparatus. GlnRS is essential for the biosynthesis 
of Gln-tRNA ${ }^{\text {Gln }}$ in Eukarya and some bacteria. However, Archaea, most bacteria, and organelles survive without GlnRS, because they encode a nondiscriminating GluRS charging tRNA Glu and tRNA ${ }^{\text {Gln }}$ with glutamate. The misacylated Glu-tRNA ${ }^{\text {Gln }}$ intermediate is then converted to Gln-tRNA ${ }^{\text {Gln }}$ by the glutamine-dependent Glu-tRNA ${ }^{\text {Gln }}$ amidotransferase, designated Glu-Adt. Thus, a nondiscriminating GluRS combined with the Glu-Adt activity is sufficient to provide the translation machinery with both amino acids, Glu and Gln. However, some bacteria, such as Helicobacter pylori, encode two essential coexisting GluRSlike enzymes, GluRS1 and GluRS2. Tamara L. Hendrickson demonstrated that these two enzymes have different substrate specificities. GluRS1 optimally aminoacylates the two tRNA $^{\text {Glu }}$ isoacceptors of $H$. pylori, whereas GluRS2 preferentially charges $H$. pylori tRNA ${ }^{\text {Gln }}$. GluRS2 may thus be a transient GluRS-like ancestor of GlnRS (termed a "GluGlnRS"), representing the missing link in the evolution from a nondiscriminating GluRS to a specific GlnRS.

A fundamental goal in the field is to understand early evolutionary events that represent committed steps in the development of the present-day protein synthesizing machinery. Koji Tamura presented experiments on RNA-directed aminoacylation of an oligonucleotide in a proteinfree system. A minihelix with the 3 '-terminal sequence ACCA was used as a tRNA mimic and incubated with a reactive 5 '-aminoacyl phosphate oligonucleotide. A prerequisite for charging of the minihelix RNA was the presence of a small guide RNA that simultaneously base-paired with the ACCA sequence of the minihelix and the aminoacyl phosphate oligonucleotide, thereby juxtaposing the reacting groups. Remarkably, Koji Tamura could show that aminoacylation was restricted to the use of L-amino acids, whereas the $\mathrm{D}$ forms were not accepted, which may simulate the point in evolution where homochirality of amino acids was determined.

Noteworthy are two in-depth enzyme kinetic analyses exploring the chemical and mechanistic basis of specific aminoacylation. Christopher S. Francklyn and coworkers, in their study on HisR, identified transfer of the amino acid to the $3^{\prime}$ end of the tRNA as the rate-limiting step, utilizing quench-flow rapid kinetic techniques. In contrast, John Perona and coworkers found out that the rate-limiting step in the cognate aminoacylation by GlnRS is product release, and not the rate of the aminoacyl transfer.

Expansion of genetic code was the main focus of the session on "Translation and Genetic Code." A natural example is the rare amino acid pyrrolysine incorporated into monomethylamine methyltransferase $(m t m B)$ of the Euryarchaeote Methanosarcina barkeri and encoded by an internal UAG termination codon. Translation of UAG requires the aminoacylation of a special amber suppressor tRNA $^{\text {Pyl }}$. Carla Polycarpo presented a study conducted to understand aminoacylation of $\mathrm{tRNA}^{\mathrm{Pyl}}$. M. barkeri contains two Lys-tRNA synthetases, one belonging to class I and the other to class II. Surprisingly, both LysRS enzymes act in concert to achieve aminoacylation of $\mathrm{tRNA}^{\mathrm{Pyl}}$. Biochemical experiments indicate that the class I and II LysRS enzymes must bind tRNA ${ }^{\mathrm{Pyl}}$ simultaneously to form a ternary complex that is able to catalyze aminoacylation. Inhibition experiments suggest that aminoacylation activity resides in the class II enzyme. The $p y l S$ gene product, originally thought to catalyze charging of $\mathrm{tRNA}^{\mathrm{Pyl}}$ with lysine, may play a role in the modification of lysine to pyrrolysine.

Site-specific insertion of unnatural amino acids into proteins via translation remains an intensively investigated topic. One strategy is the use of termination codons in connection with suppressor tRNAs. Caroline Köhrer and Uttam L. RajBhandary have expanded the concept by concomitant suppression of amber and ochre codons in a single mRNA in mammalian cells. The crux of the strategy is that the aminoacylated bacterial suppressor tRNAs are imported into the mammalian host cells and do not serve as substrate for endogenous aaRS enzymes. Potential applications include the generation of proteins with two different fluorescent residues (e.g., for FRET studies) or with phosphonylated Tyr and Thr residues (e.g., in the context of a MAP kinase). A different strategy to expand the genetic code has been pursued by Ichiro Hirao and Shigeyuki Yokoyama. Instead of exploiting the suppression of stop codons, they developed novel unnatural base pairs for nucleic acids. The two nucleobases of the pair interact via hydrogen bonding and shape complementarity just as canonical Watson-Crick base pairs do. These base pairs (termed "s:y" or "s:z") can be used for the synthesis of proteins with amino acid analogs at specific positions in a cell-free transcription-translation system. In such a setup, the novel nucleobase " $s$ " is, for example, placed at a specific location in the DNA template; the partner nucleobase " $y$ " is incorporated at complementary position during transcription by T7 RNA polymerase, and the amino acid analog is built into the nascent protein chain via an artificial tRNA that carries nucleobase " $\mathrm{s}$ " in its anticodon, thus specifically decoding the mRNA triplet with the unnatural " $y$ " base.

Aminoacyl-tRNA synthetases specifically interact with their cognate amino acids and tRNA substrates, whereas elongation factor $\mathrm{Tu}$ has to bind a spectrum of different amino acids and tRNA bodies. This raises the question of whether the ternary complexes formed between EF-Tu•GTP and different aa-tRNAs cover a broad range of complex stabilities. Olke C. Uhlenbeck and coworkers found out that evolution solved this potential problem by simply combining strongly binding amino acids with weakly binding tRNA sequences and vice versa. This principle of "thermodynamic compensation" seems to be implemented as well for tRNA binding to the ribosomal A and P sites. A conclusion from these findings is that the apparent need for thermodynamic compensation has driven tRNA sequence diversity during evolution, with sequence differences in the acceptor and $\mathrm{T}$ stem compensating for variation of amino acid affinity to 
EF-Tu, and sequence variation in other parts of the tRNA body potentially compensating for differences in codonanticodon strength.

The immense progress in X-ray analysis of ribosomal particles, unimaginable 10 years ago, has greatly improved our molecular understanding of how the ribosome senses cognate tRNA codon-anticodon interaction and how aminoglycoside antibiotics specifically interfere with this process. The ribosomal A site switches between two conformational states, with two adenines "looped out" in one but not the other state. The looped-out state is induced by a correct codon-anticodon interaction, but also by aminoglycosides causing the observed losses of translation fidelity. Eric Westhof presented the molecular details of the ribosomal A site structure in the context of its interaction with tRNA, mRNA, and antibiotics, leaving us with an important take-home message: The most promising RNA target motifs for novel antibacterial compounds will be those that undergo a dynamical exchange between discrete alternative conformations (molecular switches).

Thomas Steitz, in his lecture sponsored by the "Otto Warburg Chemie-Stiftung," updated us on one of the central themes of the meeting, the dissection of the catalytic mechanism of peptide bond formation at the large ribosomal subunit. The experimental strategy has been X-ray analysis of $50 \mathrm{~S}$ ribosomal subunits in complex with various substrate and product analogs of the CCA end of tRNA bound to the A, P, or E site, including a new transition state analog. Regarding tRNA E-site binding, cocrystal structures of Haloarcula marismortui 50S subunits and two different mimics of E-site-bound tRNA have revealed that this site sterically excludes aa-tRNA, thus explaining its specificity for deacylated tRNA.

Mikel Valle presented the cryo-EM-derived models for conformational changes associated with the decoding process and the codon recognition-dependent GTPase reaction catalyzed by EF-Tu, one of the open key questions concerning the mechanism of precise codon-anticodon recognition. Combination of information from cryo-EM and X-ray crystallography appears the most promising strategy for the way to understand the structural states of tRNA on the ribosome. The detailed kinetic studies on aminoacyl-tRNA selection by programmed ribosomes as presented by Marina Rodnina will, however, remain the only basis for the functional and kinetic interpretation of the conformational states identified by structural studies. A central focus of $\mathrm{M}$. Rodnina and coworkers is to understand how the events at the decoding center on the $30 \mathrm{~S}$ subunit are transmitted to the GTPase and peptidyl transferase centers on the 50S subunit. Here, streptomycin is used as a tool that arrests the ribosome in a conformation characterized by slow communication between subunits and nonselective treatment of cognate versus near-cognate codon-anticodon interactions.

Nenad Ban presented the recently determined structure of the tRNA-like domain of tmRNA in complex with small protein B (SmpB), a protein cofactor essential for biological function of tmRNA. The most striking features of this structure are the unusual position of the T $\Psi \mathrm{C}$ arm and a larger angle between the equivalents to anticodon and acceptor arm. The structure permitted formulation of a model of tmRNA binding on the ribosome. Apart from the unique function of tmRNA, these findings could suggest that the canonical L-shaped tRNA structure might not be maintained during all steps of a regular ribosomal elongation cycle.

How the aminoacylation status of tRNA regulates gene expression and induces the stringent response in bacteria was the topic of contributions by Måns Ehrenberg and Daniel N. Wilson, respectively. Daniel N. Wilson and coworkers illuminated the old stringent response ("magic spot") mystery by dissecting the functional properties of the (p)ppGpp synthesis factor RelA. M. Ehrenberg presented a theoretical approach developed to predict the effects of different aminoacylation levels of tRNA isoacceptors when the cognate amino acid becomes growth limiting. The basic idea is that whenever an amino acid is attached to different tRNA isoacceptors reading different codons, shortage of this amino acid will result in the lowest charging level for isoacceptors with the lowest ratio of tRNA concentration to codon frequency. The theory, supported by experimental data from the E. coli system, explains biased codon usage for the cognate amino acid in some leader regions and structural genes of amino acid biosynthetic operons, such as the thr operon.

The rapid progress in the structural determination of components of the translational apparatus has also shed new light on the mechanism of translation termination, stimulating the formulation of new concepts and challenging old ideas. Ludmila Frolova presented her view on the function of release factors and provided further evidence that allows us to look at these proteins as functional analogs of aminoacyl-tRNAs that interact with the ribosomal A site.

How some animal mitochondrial tRNAs with unusual structures, for example, those missing a T or D arm, can function in animal mitochondrial protein synthesis has long been a mystery. The systematic work on the mammalian mitochondria, translation apparatus, performed for more than 20 years in Kimitsuna Watanabe's laboratory, is increasingly rewarded with the fruits of understanding. Several mitochondrial tRNA modifications have been identified. The mitochondrial translation system was reconstituted in vitro and its components interchanged systematically with the components of bacterial translation systems. As a result we start to comprehend the differences between bacterial and mitochondrial translation, an important step toward the understanding of the molecular causes of human mitochondria-related diseases, a central theme in the first part of this meeting. K. Watanabe and coworkers have recently identified two distinct EF-Tu proteins in nematode mitochondria, named EF-Tu1 and EF-Tu2, 
which differ from bacterial EF-Tu by C-terminal extensions. The two EF-Tu proteins were found to be specific for tRNAs lacking the T arm (EF-Tu1) or tRNA ${ }^{\text {Ser }}$ species missing the D arm (EF-Tu2). Biochemical experiments revealed that EF-Tul interacts with the $\mathrm{D}$ arm in addition to the acceptor stem, representing a gain of function compared with bacterial EF-Tu and suggesting that the C-terminal extension is involved in D arm recognition by EF-Tul.

Our overview of the meeting in Banz must remain in- complete and fragmentary because a plethora of interesting talks and poster contributions (more than 150) could not be appreciated due to space limitations. We hope that many aspects of the promising research, predominantly presented by a large number of young participants, will get the chance to be presented as a lecture in the next meeting, two years from now. The 21st "tRNA World" meeting will be hosted in December 2005 by the Indian Institute of Science in Bangalore, India. 

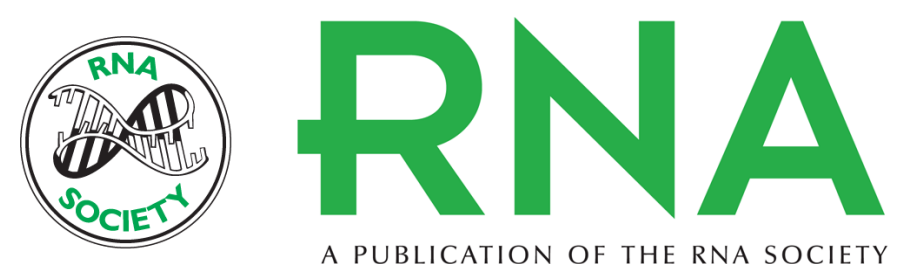

A PUBLICATION OF THE RNA SOCIETY

\section{The tRNA world}

ROLAND K. HARTMANN, MARIO MÖRL and MATHIAS SPRINZL

RNA 2004 10: 344-349

\section{License}

Email Alerting Receive free email alerts when new articles cite this article - sign up in the box at the Service top right corner of the article or click here. 